\title{
Black hole formation in the Friedmann universe: Formulation and computation in numerical relativity
}

\author{
Masaru Shibata ${ }^{1,2}$ and Misao Sasaki ${ }^{1,3}$ \\ ${ }^{1}$ Department of Earth and Space Science, Graduate School of Science, Osaka University, \\ Toyonaka, Osaka 560-0043, Japan \\ ${ }^{2}$ Department of Physics, University of Illinois at Urbana-Champaign, Urbana, Il 61801 \\ ${ }^{3}$ Department of Physics, School of Science, University of Tokyo, Bunkyoku, Tokyo 113-0033, Japan
}

\begin{abstract}
We study formation of black holes in the Friedmann universe. We present a formulation of the Einstein equations under the constant mean curvature time-slicing condition. Our formalism not only gives us the analytic solution of the perturbation equations for non-linear density and metric fluctuations on superhorizon scales, but also allows us to carry out a numerical relativity simulation for black hole formation after the scale of the density fluctuations is well within the Hubble horizon scale. We perform a numerical simulation of spherically symmetric black hole formation in the radiation-dominated, spatially flat background universe for a realistic initial condition supplied from the analytic solution. It is found that the initial metric perturbation has to be non-linear (the maximum value of $3 \mathrm{D}$ conformal factor $\psi_{0}$ at $t=0$ should be larger than $\sim 1.4$ ) for a black hole to be formed, but the threshold amplitude for black hole formation and the final black hole mass considerably depend on the initial density (or metric) profile of the perturbation: The threshold value of $\psi_{0}$ at $t=0$ for formation of a black hole is smaller for a high density peak surrounded by a low density region than for that surrounded by the average density region of the flat universe. This suggests that it is necessary to take into account the spatial correlation of density fluctuations in the study of primordial black hole formation.
\end{abstract}

PACS: 04.25.Dm, 95.35.+d, 97.60.Lf

\section{INTRODUCTION}

The formation of black holes in the early universe and its cosmological implications have been discussed in a variety of contexts for decades [1]. However, it has long been thought that it would be practically impossible to prove or disprove the existence of these primordial black holes. Recent discoveries of microlensing events by MACHOs of mass $\sim 0.5 M_{\odot}$ in the halo of our galaxy [2] have dramatically changed this situation. By various other means of observation, it may be possible to deny all the other possibilities and hence to identify MACHOs with black holes. Then these MACHO black holes must be primordial since it is impossible to form a black hole of mass smaller than $\sim M_{\odot}$ as a result of stellar evolution [3]. Furthermore, it has been recently suggested that if MACHOs are in fact primordial black holes, the number of binaries that are just coalescing today may be large enough to be directly detected by the oncoming gravitational wave observatories such as LIGO, VIRGO, GEO and TAMA within a few years 谏. Consequently, it has become an urgent issue to quantify how and when these black holes could be formed in a precise manner.

Among other possibilities, primordial black holes are most conceivably formed from large curvature perturbations generated during an inflationary stage of the very early universe [0.6]. The curvature perturbations generated in the inflationary universe are dominated by the so-called growing adiabatic mode of density perturbations. In the linear theory, the evolution of these perturbations is well studied and their temporal behavior is known throughout the whole stage from the epoch when their wavelengths are much larger than the Hubble horizon scale until their evolution becomes non-linear on scales much smaller than the Hubble horizon.

However, the amplitude must be already large enough (of order unity) to form black holes when the characteristic wavelengths of the perturbations were on superhorizon scales. Furthermore, the formation of a black hole is itself a fully general relativistic phenomenon. The evolution of non-linear density perturbations on superhorizon scales was investigated by several authors and the threshold amplitude of curvature perturbations on superhorizon scales for forming black holes was estimated [7, 8]. These previous estimates of the threshold amplitude were based on approximate analytical treatments and/or on rather naive numerical simulations, hence are admittedly crude. Furthermore, there is a crucial reason that requires us an accurate estimate as follows: According to the inflationary scenario, the probability distribution of the curvature perturbations is essentially Gaussian and primordial black holes are produced from the high amplitude tail of the distribution. Therefore, a small error in the estimate of the threshold amplitude will result in a large error in that of the number of produced black holes. Thus threshold amplitude must be estimated accurately. 
As a first step to accomplish this purpose, in this paper, we present a new formalism by which it is possible to follow the formation of a primordial black hole throughout the whole stage starting from the very early universe when the perturbation is well outside the Hubble horizon to the final stage when a black hole is formed. More specifically, our formalism not only gives us the analytic solution of non-linear curvature perturbations on superhorizon scales but also allows us to perform a numerical simulation of the black hole formation with the initial data given by the analytic solution, with no need of changing the gauge conditions or of numerical matching. In addition, it may be worthwhile to mention that the constant mean curvature time-slicing employed here is equivalent to the so-called constant Hubble slicing in cosmological perturbation theory [9]. And, it has been pointed out that the constant Hubble slicing is most appropriate for evaluating non-linear curvature fluctuations generated during inflation [10]. Hence the initial curvature perturbation spectrum evaluated in models of inflation can be directly used for the initial data of our problem.

Then using our formalism, we carry out a spherically symmetric simulation of black hole formation in the radiation-dominated Friedmann universe. We consider the initial data with two parameters; one describes the amplitude and the other the radial profile. We find that both the threshold amplitude for black hole formation and the final black hole mass depend appreciably on the initial profile of the perturbation. We also consider another possible criterion for black hole formation by defining a compaction function of the perturbation profile. Although this function can be defined only for a spherically symmetric configuration, we find the maximum value of this function gives us a better criterion for the formation of black holes.

While this paper was in preparation, a couple of papers on the primordial black hole formation appeared on the astro-ph 11]. It seems that their formalism is powerful for studying the formation of a black hole in a spherical symmetric spacetime. However, it does not seem convenient to give a realistic initial condition which should be supplied just after inflation. Actually, they give initial conditions at the epoch when the scale of the density fluctuation is as small as the Hubble horizon scale. Since the density fluctuation is already nonlinear at that epoch, it is impossible to control the initial date so that it reduces to the growing adiabatic mode when the evolution is traced back in time to the very early universe. In other words, their initial conditions are inevitably contaminated by unrealistic decaying mode perturbations which badly diverge as $t \rightarrow 0$. As a result, though the criterion for black hole formation they find is new and interesting, it cannot be directly related to the initial condition at the end of inflation. So that it is not convenient for a practical study of primordial black hole formation. Furthermore, application of their formalism is restricted to the spherical symmetric case (i.e., very special case). In contrast, in ours, it is easy to relate a criterion of black hole formation to an initial condition just after inflation, and also it can be applied to general $3 \mathrm{D}$ cases. The only restriction of our formalism is that the spacetime be asymptotically spatially flat Friedmann.

The paper is organized as follows. In Sec. II, we present the Einstein and hydrodynamic equations in the Friedmann universe using the $3+1$ formalism, which have appropriate forms for numerical relativity simulations. We then introduce the constant mean curvature timeslicing in which the equations for geometric variables have similar forms to those in the maximal slice condition in the asymptotically flat spacetime. In Sec. III, assuming that the length scale of a density fluctuation is always much longer than the Hubble horizon scale, we take the long wavelength limit of the equations derived in Sec. II, and then find the analytic solution for the perturbation equations. In Sec. IV, we perform numerical simulations of black hole formation in a spherically symmetric, radiation-dominated universe using initial conditions given by the analytic solution in Sec. III. Sec. V is devoted to summary. Throughout this paper, we use the units $c=1=G$.

\section{FORMULATION}

We present the Einstein and hydrodynamic equations in the Friedmann universe using the $3+1$ formalism in general relativity. We write the line element as

$$
\begin{aligned}
d s^{2} & =g_{\mu \nu} d x^{\mu} d x^{\nu} \\
& =\left(-\alpha^{2}+\beta_{k} \beta^{k}\right) d t^{2}+2 \beta_{i} d x^{i} d t+\gamma_{i j} d x^{i} d x^{j}
\end{aligned}
$$

where $g_{\mu \nu}, \alpha, \beta^{i}\left(\beta_{i}=\gamma_{i j} \beta^{j}\right)$, and $\gamma_{i j}$ are the 4D metric, lapse function, shift vector, and $3 \mathrm{D}$ spatial metric, respectively. Since we consider an asymptotically spatially flat Friedmann universe, we rewrite $\gamma_{i j}$ as

$$
\gamma_{i j}=\psi^{4} a(t)^{2} \tilde{\gamma}_{i j}
$$

and impose the condition $\operatorname{det}\left(\tilde{\gamma}_{i j}\right)=\operatorname{det}\left(\eta_{i j}\right) \equiv \eta$, where $\eta_{i j}$ is a flat spatial metric. Here, $a(t)$ is defined to be the scale factor in the homogeneous universe, i.e., the scale factor in the asymptotic region, and we determine it from the well known equations for the scale factor as

$$
\begin{aligned}
& \ddot{a}=-\frac{4 \pi}{3} a\left[\rho_{0}(t)+3 P_{0}(t)\right], \\
& \dot{a}^{2}=\frac{8 \pi}{3} a^{2} \rho_{0}(t)
\end{aligned}
$$

where $\rho_{0}$ and $P_{0}$ are the density and pressure for the homogeneous universe, and $\dot{a}=\partial_{t} a$. As we find below, $a_{0}$, $\rho_{0}$ and $P_{0}$ are automatically determined when an equation of state is provided.

We also rewrite the extrinsic curvature $K_{i j}$ as

$$
K_{i j}=\psi^{4} a^{2} \tilde{A}_{i j}+\frac{\gamma_{i j}}{3} K
$$


where $K=K_{k}{ }^{k}$ and hence $\tilde{A}_{i j}$ is defined to be traceless. The indices of $\tilde{A}_{i j}$ and $\tilde{A}^{i j}$ are to be raised or lowered in terms of $\tilde{\gamma}^{i j}$ and $\tilde{\gamma}_{i j}$. In numerical computation, we will solve $\tilde{\gamma}_{i j}, \tilde{A}_{i j}, \psi$ and $K$ instead of $\gamma_{i j}$ and $K_{i j}$. Hereafter, we use $D_{i}$ and $\tilde{D}_{i}$ as the covariant derivatives with respect to $\gamma_{i j}$ and $\tilde{\gamma}_{i j}$, respectively.

As a source of the energy momentum tensor, we consider a perfect fluid for which the energy momentum tensor is written as

$$
T_{\mu \nu}=(\rho+P) u_{\mu} u_{\nu}+P g_{\mu \nu},
$$

where $\rho, P$ and $u^{\mu}$ are the energy density, pressure and four velocity, respectively. Hereafter, we assume a relativistic polytropic equation of state, $P=(\Gamma-1) \rho$, where $\Gamma$ is a polytropic constant and we assume $\Gamma>1$ in the following discussion. For the relativistic polytropic equation of state, we get the following relations from Eqs. (2.4)

$$
a=a_{f} t^{2 / 3 \Gamma} \text { and } \rho_{0}=\frac{1}{6 \pi \Gamma^{2} t^{2}},
$$

where $a_{f}$ is a constant.

The hydrodynamic equations are written in the form

$$
\begin{gathered}
\partial_{t}\left(w \psi^{6} a^{3} \rho^{1 / \Gamma}\right)+\frac{1}{\eta^{1 / 2}} \partial_{k}\left(\eta^{1 / 2} w \psi^{6} a^{3} \rho^{1 / \Gamma} v^{k}\right)=0 \\
\partial_{t}\left\{w \psi^{6} a^{3}(\rho+P) u_{j}\right\}+\frac{1}{\eta^{1 / 2}} \partial_{k}\left\{\eta^{1 / 2} w \psi^{6} a^{3}(\rho+P) v^{k} u_{j}\right\} \\
=-\alpha \psi^{6} a^{3} \partial_{j} P+w \psi^{6} a^{3}(\rho+P)\left\{-\alpha u^{0} \partial_{j} \alpha\right. \\
\left.+u_{k} \partial_{j} \beta^{k}-\frac{u_{k} u_{l}}{2 u^{0}} \partial_{j} \gamma^{k l}\right\}
\end{gathered}
$$

where $w \equiv \alpha u^{0}$, and

$$
v^{k} \equiv \frac{u^{k}}{u^{0}}=-\beta^{k}+\tilde{\gamma}^{k l} \frac{u_{l}}{\psi^{4} a^{2} u^{0}} .
$$

Evolution equations for geometric variables are written as follows 12]:

$$
\begin{aligned}
\left(\partial_{t}-\beta^{k} \partial_{k}\right) \tilde{\gamma}_{i j}= & -2 \alpha \tilde{A}_{i j} \\
+ & \tilde{\gamma}_{i k} \beta^{k}{ }_{, j}+\tilde{\gamma}_{j k} \beta^{k}{ }_{, i}-\frac{2}{3} \tilde{\gamma}_{i j(0)} D_{k} \beta^{k}, \\
\left(\partial_{t}-\beta^{k} \partial_{k}\right) \tilde{A}_{i j}= & \frac{1}{a^{2} \psi^{4}}\left[\alpha\left(R_{i j}-\frac{\gamma_{i j}}{3} R\right)\right. \\
& \left.\quad\left(D_{i} D_{j} \alpha-\frac{\gamma_{i j}}{3} D_{k} D^{k} \alpha\right)\right] \\
& +\alpha\left(K \tilde{A}_{i j}-2 \tilde{A}_{i k} \tilde{A}_{j}{ }^{k}\right) \\
& +\beta_{, i}^{k} \tilde{A}_{k j}+\beta^{k}{ }_{, j} \tilde{A}_{k i}-\frac{2}{3}{ }_{(0)} D_{k} \beta^{k} \tilde{A}_{i j} \\
& -\frac{8 \pi \alpha}{a^{2} \psi^{4}}\left(S_{i j}-\frac{\gamma_{i j}}{3} S_{k}{ }^{k}\right) \\
\left(\partial_{t}-\beta^{k} \partial_{k}\right) \psi+ & \frac{\dot{a}}{2 a} \psi=\frac{\psi}{6}\left\{-\alpha K+{ }_{(0)} D_{k} \beta^{k}\right\} \\
\left(\partial_{t}-\beta^{k} \partial_{k}\right) K= & \alpha\left(\tilde{A}_{i j} \tilde{A}^{i j}+\frac{1}{3} K^{2}\right) \\
& -D_{k} D^{k} \alpha+4 \pi \alpha\left(E+S_{k}{ }^{k}\right)
\end{aligned}
$$

where $R_{i j}$ is the Ricci tensor with respect to $\gamma_{i j},{ }_{(0)} D_{k}$ is the covariant derivative with respect to $\eta_{i j}$, and

$$
S_{i j}=(\rho+P) u_{i} u_{j}+P \gamma_{i j} .
$$

To clarify the meaning of equation (2.12), we rewrite $R_{i j}$ as

$$
R_{i j}=\tilde{R}_{i j}+R_{i j}^{\psi}
$$

where $\tilde{R}_{i j}$ is the Ricci tensor with respect to $\tilde{\gamma}_{i j}$ and

$$
\begin{aligned}
R_{i j}^{\psi}= & -\frac{2}{\psi} \tilde{D}_{i} \tilde{D}_{j} \psi-\frac{2}{\psi} \tilde{\gamma}_{i j} \tilde{\Delta} \psi \\
& +\frac{6}{\psi^{2}} \tilde{D}_{i} \psi \tilde{D}_{j} \psi-\frac{2}{\psi^{2}} \tilde{\gamma}_{i j} \tilde{D}_{k} \psi \tilde{D}^{k} \psi
\end{aligned}
$$

$\tilde{R}_{i j}$ is written as

$$
\begin{aligned}
\tilde{R}_{i j}=\frac{1}{2}[ & -{ }_{(0)} \Delta \tilde{\gamma}_{i j}+{ }_{(0)} D_{j(0)} D^{k} \tilde{\gamma}_{k i}+{ }_{(0)} D_{i(0)} D^{k} \tilde{\gamma}_{k j} \\
& \left.+2_{(0)} D_{k}\left(f^{k l} C_{l, i j}\right)-2 C_{k j}^{l} C_{i l}^{k}\right],
\end{aligned}
$$

where ${ }_{(0)} \Delta$ is the Laplacian with respect to $\eta_{i j}, f^{k l}=$ $\tilde{\gamma}^{k l}-\eta^{k l}$, and

$$
C_{i j}^{k}=\frac{\tilde{\gamma}^{k l}}{2}\left({ }_{(0)} D_{i} \tilde{\gamma}_{j l}+{ }_{(0)} D_{j} \tilde{\gamma}_{i l}-{ }_{(0)} D_{l} \tilde{\gamma}_{i j}\right) .
$$

Note that we use a relation $\tilde{\gamma}^{i j}{ }_{(0)} D_{k} \tilde{\gamma}_{i j}=\eta^{i j}{ }_{(0)} D_{k} \eta_{i j}=0$ to derive Eq. (2.18). From Eq. (2.18), it is found that under an appropriate gauge condition such as a transversetraceless (TT) gauge, ${ }_{(0)} D^{k} \tilde{\gamma}_{k j}=0$, Eqs. (2.11) and (2.12) are found to constitute a wave equation for tensor $\tilde{\gamma}_{i j}$.

Hamiltonian and momentum constraint equations are

$$
\begin{aligned}
& R_{k}{ }^{k}-\tilde{A}_{i j} \tilde{A}^{i j}+\frac{2}{3} K^{2}=16 \pi E, \\
& D_{i} \tilde{A}^{i}{ }_{j}-\frac{2}{3} D_{j} K=8 \pi J_{j},
\end{aligned}
$$

where

$$
\begin{aligned}
& E=(\rho+P) w^{2}-P, \\
& J_{i}=(\rho+P) w u_{i},
\end{aligned}
$$

We may write the constraint equations as

$$
\begin{aligned}
& \tilde{\Delta} \psi= \frac{\tilde{R}_{k}^{k}}{8} \psi-2 \pi \psi^{5} a^{2} E \\
&-\frac{\psi^{5} a^{2}}{8}\left(\tilde{A}_{i j} \tilde{A}^{i j}-\frac{2}{3} K^{2}\right), \\
& \tilde{D}^{j}\left(\psi^{6} \tilde{A}_{i j}\right)-\frac{2}{3} \psi^{6} \tilde{D}_{i} K=8 \pi J_{i} \psi^{6},
\end{aligned}
$$

where $\tilde{\Delta}$ is the Laplacian with respect to $\tilde{\gamma}_{i j}$. 
In this paper, we choose a constant mean curvature slice as we chose in a previous paper 13.

$$
K=K(t)=-\frac{3 \dot{a}}{a} .
$$

This choice can most effectively factor out overall factors of the expansion of the background universe from the dynamical variables. In this case, we obtain the equation for $\alpha$ as

$$
\begin{aligned}
\Delta \alpha=\alpha[ & 4 \pi\left\{2(\rho+P)\left(w^{2}-1\right)+\rho-\rho_{0}+3\left(P-P_{0}\right)\right\} \\
+ & \left.\tilde{A}_{i j} \tilde{A}^{i j}\right]+12 \pi\left(\rho_{0}+P_{0}\right)(\alpha-1),
\end{aligned}
$$

where $\Delta$ is the Laplacian with respect to $\gamma_{i j}$. We also note that Eqs. 2.13) and (2.24) are, respectively, rewritten as

$$
\begin{gathered}
\left(\partial_{t}-\beta^{k} \partial_{k}\right) \psi=\frac{\dot{a}}{2 a} \psi(\alpha-1)+\frac{\psi}{6 \eta^{1 / 2}}\left(\eta^{1 / 2} \beta^{k}\right)_{, k} \\
\tilde{\Delta} \psi=\frac{\tilde{R}_{k}^{k}}{8} \psi-2 \pi \psi^{5} a^{2}\left[(\rho+P)\left(w^{2}-1\right)+\rho-\rho_{0}\right] \\
-\frac{\psi^{5} a^{2}}{8} \tilde{A}_{i j} \tilde{A}^{i j} .
\end{gathered}
$$

Thus, in the constant mean curvature slice condition, the equations for $\alpha$ and $\psi$ are similar to those for the maximal slice condition, $K=0$, in the asymptotically flat spacetime. Hence, we expect that it has a singularity avoidance property for the case of black hole formation.

\section{LONG WAVELENGTH LIMIT}

In this section, we consider the so-called long wavelength approximation assuming that the characteristic length scale $L$ of a density fluctuation is always much larger than the Hubble horizon scale, $L \gg t \sim a / \dot{a}$. First, we introduce a small parameter $\epsilon$, and assume that $\delta \equiv \rho / \rho_{0}-1$ is of $O\left(\epsilon^{2}\right)$ and its characteristic length scale is of $O(1 / \epsilon)$. The latter assumption is equivalent to assuming that the magnitude of spatial gradients of the quantities is given by $\partial_{i} \psi=\psi \times O(\epsilon), \partial_{i} \alpha=\alpha \times O(\epsilon)$, $\partial_{i} \delta=\delta \times O(\epsilon)$ and so on. Then, it is found from the equations presented in Sec. II that the following relation should hold:

$$
\begin{aligned}
& \psi-1=O\left(\epsilon^{0}\right), \\
& u^{i}=O\left(\epsilon^{1}\right), \\
& \tilde{A}_{i j}, h_{i j}\left(\equiv \tilde{\gamma}_{i j}-\eta_{i j}\right), \chi(\equiv \alpha-1), \delta=O\left(\epsilon^{2}\right), \\
& u_{i}, v^{i}+\beta^{i}=O\left(\epsilon^{3}\right) .
\end{aligned}
$$

Here we have assumed for simplicity that the amplitude of primordial gravitational wave perturbations is negligible. We note that because the gravitational wave perturbations do not decay with time evolution, the following solution can change considerably if their amplitude is not small initially. We have also assumed that the vector part is absent for any quantity; in other words, we do not consider vorticity.

It is worth mentioning that these assumptions are naturally realized in most of successful inflation models. In the inflationary universe scenario, only the so-called growing mode perturbations of scalar and tensor types survive and amplitude of the tensor perturbation is generally very small. We note that $\psi-1$ may be larger than unity, i.e., we have not used any approximation for treating $\psi$. On the other hand, other quantities should be small enough and are regarded as small perturbations. If $\psi-1 \ll 1$ (i.e., if the linear theory applies), the above corresponds to assuming the existence of only the adiabatic growing mode.

Because we have not yet imposed any condition on $\beta^{k}$, we cannot specify the order of magnitude of $\beta^{k}$ and $v^{k}$. For example, in the case of the minimum distortion gauge [14], they are of $O(\epsilon)$. On the other hand, in the $\beta^{k}=0$ gauge, $v^{k}=O\left(\epsilon^{3}\right)$. In the following discussion, we do not have to specify the gauge condition for $\beta^{k}$ in order to obtain solutions except for $\beta^{k}$ and $v^{k}$.

Substituting the lowest order terms in $O(\epsilon)$ of each variables shown in Eqs. (3.1), we have the following equations:

$$
\begin{aligned}
& \frac{1}{\Gamma} \dot{\delta}+\frac{6 \dot{\psi}}{\psi}+\nabla_{k} v^{k}=O\left(\epsilon^{4}\right), \\
& \partial_{t}\left\{\rho_{0} a^{5} \nabla_{k}\left(v^{k}+\beta^{k}\right)\right\}+\frac{6 \dot{\psi}}{\psi} \rho_{0} a^{5} \nabla_{k}\left(v^{k}+\beta^{k}\right) \\
& +\rho_{0} a^{5} \nabla_{k}\left\{\frac{4 \dot{\psi}}{\psi}\left(v^{k}+\beta^{k}\right)\right\} \\
& =-\rho_{0} a^{3}\left(\nabla^{2} \chi+\frac{\Gamma-1}{\Gamma} \nabla^{2} \delta\right)+O\left(\epsilon^{4}\right), \\
& \frac{6 \dot{\psi}}{\psi}-3 \frac{\dot{a}}{a} \chi=\nabla_{k} \beta^{k}+O\left(\epsilon^{4}\right), \\
& { }_{(0)} \Delta \psi=-2 \pi a^{2} \psi^{5} \rho_{0} \delta+O\left(\epsilon^{4}\right) \text {, } \\
& \nabla^{2} \chi=4 \pi \rho_{0} a^{2}\{(3 \Gamma-2) \delta+3 \Gamma \chi\}+O\left(\epsilon^{6}\right), \\
& \partial_{t} h_{i j}=-2 \tilde{A}_{i j}+\delta_{i k} \beta_{, j}^{k}+\delta_{j k} \beta_{, i}^{k}-\frac{2}{3} \delta_{i j} \beta_{, k}^{k} \\
& +O\left(\epsilon^{4}\right) \\
& \partial_{t} \tilde{A}_{i j}+3 \frac{\dot{a}}{a} \tilde{A}_{i j} \\
& =\frac{1}{\psi^{4} a^{2}}\left[-\frac{2}{\psi}\left({ }_{(0)} D_{i(0)} D_{j} \psi-\frac{\eta_{i j}}{3}{ }_{(0)} \Delta \psi\right)\right. \\
& \left.+\frac{6}{\psi^{2}}\left({ }_{(0)} D_{i} \psi_{(0)} D_{j} \psi-\frac{\eta_{i j}}{3}{ }_{(0)} D_{k} \psi_{(0)} D_{k} \psi\right)\right] \\
& +O\left(\epsilon^{4}\right) \text {, }
\end{aligned}
$$

where

$$
\begin{aligned}
\nabla_{k} & =\frac{1}{\psi^{6} \eta^{1 / 2}} \partial_{k} \psi^{6} \eta^{1 / 2} \\
\nabla^{2} & =\frac{1}{\psi^{6} \eta^{1 / 2}} \partial_{k}\left(\psi^{2} \eta^{1 / 2} \eta^{k l} \partial_{l}\right)
\end{aligned}
$$


The first two equations, 3.2) and (3.3), are derived from hydrodynamic equations and other five, (3.4)-(3.8), are derived from equations for geometric variables.

From Eqs. (3.2) and (3.4), we find that the following relations have to hold;

$$
\begin{aligned}
& \dot{\psi}=O\left(\epsilon^{2}\right), \\
& \frac{1}{\Gamma} \dot{\delta}+3 \frac{\dot{a}}{a} \chi=-\nabla_{k}\left(v^{k}+\beta^{k}\right)=O\left(\epsilon^{4}\right) .
\end{aligned}
$$

Also, we find that the right-hand side of Eq. (3.6) has to be of $O\left(\epsilon^{4}\right)$, i.e.,

$$
(3 \Gamma-2) \delta+3 \Gamma \chi=O\left(\epsilon^{4}\right) .
$$

From these relations and a reasonable assumption that $\delta \rightarrow 0$ for $t \rightarrow 0$, we finally find the time dependence for each variable at the leading order in $O(\epsilon)$ as

$$
\begin{aligned}
& \delta=-\frac{3 \Gamma}{3 \Gamma-2} \chi \propto t^{2-4 / 3 \Gamma} \\
& \nabla_{k}\left(v^{k}+\beta^{k}\right) \propto t^{3-8 / 3 \Gamma} \\
& u_{k} \propto t^{3-4 / 3 \Gamma} \\
& \psi \propto t^{0} \\
& \tilde{A}_{i j} \propto t^{1-4 / 3 \Gamma}
\end{aligned}
$$

Time dependence of $v^{i}, \beta^{i}, O\left(\epsilon^{2}\right)$ part of $\psi$, and $h_{i j}$ is found when we give spatial gauge condition for $\beta^{i}$. For example, in the TT gauge and/or minimum distortion gauge [14], it is found that

$$
\begin{aligned}
& v^{k}, \beta^{k} \propto t^{1-4 / 3 \Gamma}, \\
& h_{i j} \propto t^{2-4 / 3 \Gamma} \\
& O\left(\epsilon^{2}\right) \text { part of } \psi \propto t^{2-4 / 3 \Gamma} .
\end{aligned}
$$

We emphasize again that we do not restrict our attention to the case where $\psi-1 \ll 1$. Namely, even when the scalar part of the metric is non-linear, we can still find the analytic solution as long as the long wavelength approximation holds.

The purpose of this paper is to give a framework to investigate the primordial black hole formation process, and its standard formation scenario is as follows: In a very early phase of the universe, just after inflation, the scalar-type perturbations generated from the quantum fluctuations of an inflaton field have the length scale much larger than the Hubble horizon scale at that time. Some of these perturbations may have a large metric perturbation amplitude [5]. As long as its scale is larger than the Hubble horizon scale, it never collapses, but once the scale becomes smaller than the Hubble horizon scale, it collapses to form a black hole.

The advantage of our present formalism is as follows: Once we give a realistic initial condition at the very early epoch just after inflation, evolutions of the metric and density fluctuations can be analytically calculated as long as the length scale of the fluctuation is much larger than the Hubble horizon scale. Then we may start a numerical simulation sufficiently before the scale enters the Hubble horizon and follow the black hole formation process without changing the gauge condition or numerical matching of initial data. Hence, we can consistently investigate the evolution of the metric and density fluctuations throughout the whole dynamical range starting from a very early epoch of the universe at which analysis can be performed in an analytical manner up to the formation of a black hole when analysis should be done in numerical relativity.

\section{NUMERICAL STUDY IN THE SPHERICAL SYMMETRIC CASE}

To demonstrate the usefulness and robustness of our formalism, in this section, we perform numerical simulations of primordial black hole formation assuming spherical symmetry.

\section{A. Basic equations}

For the spherical case, the line element can be written as

$$
\begin{aligned}
d s^{2} & =-\left(\alpha^{2}-\psi^{4} \beta^{2} r^{2}\right) d t^{2}+2 \psi^{4} a^{2} \beta r d r d t \\
& +\psi^{4} a^{2}\left(d r^{2}+r^{2} d \Omega\right)
\end{aligned}
$$

where $\beta$ denotes $\beta^{r} / r$. We may say that we choose the minimum distortion gauge condition in this line element

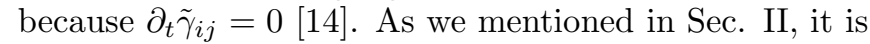
not always necessary to take the spatial gauge condition used here, and we may use other gauge conditions such as $\beta^{r}=0$. The spatial gauge condition we choose here is only one example 17 .

Since gravitational waves are not generated in the spherically symmetric spacetime, we need not solve the evolution equations for the geometrical variables if we solve the constraint equations and the equations of the gauge condition. On the other hand, if we solve the evolution equations, we can use the constraint equations in order to check the accuracy of numerical solutions in each time step. Thus, we solve the evolution equation for $\psi$ instead of the Hamiltonian constraint equation, but use the latter to check the numerical accuracy. Then, the equations for the geometric variables solved in numerical computation are as follows:

$$
\begin{aligned}
& \left(\partial_{t}-2 \beta y \partial_{y}\right) \psi=\frac{\dot{a}}{2 a} \psi(\alpha-1)+\frac{\psi}{6}\left(3 \beta+2 y \partial_{y} \beta\right), \\
& { }_{(0)} \Delta \zeta=\zeta \psi^{4} a^{2}\left[6 \pi(\rho+P)\left(w^{2}-1\right)\right. \\
& +2 \pi\left\{\rho-\rho_{0}+6\left(P-P_{0}\right)\right\} \\
& \left.+12 \pi\left(\rho_{0}+P_{0}\right)+\frac{21}{16} A^{2}\right] \\
& +\psi^{5} a^{2}\left[4 \pi \left\{2(\rho+P)\left(w^{2}-1\right)\right.\right.
\end{aligned}
$$




$$
\begin{aligned}
& \left.\left.+\rho-\rho_{0}+3\left(P-P_{0}\right)\right\}+\frac{3}{2} A^{2}\right], \\
& y \partial_{y} \beta=\frac{3}{4} \alpha A, \\
& \partial_{r}\left(r^{3} \psi^{6} A\right)=8 \pi r^{4} \psi^{6}(\rho+P) w u,
\end{aligned}
$$

where $y=r^{2}, u=u_{r} / r$ and $\zeta=\psi(\alpha-1)$. We use the relation $-\tilde{A}_{\theta}{ }^{\theta} / 2=-\tilde{A}_{\varphi}^{\varphi} / 2=\tilde{A}_{r}^{r} \equiv A$. Eq. (4.3) is solved under the boundary condition at $r \rightarrow \infty$ as

$$
\zeta=\frac{C}{r} e^{-r / r_{0}}+O\left(r^{-2}\right),
$$

where $C$ is a constant, and $r_{0}=1 / \sqrt{12 \pi \Gamma \rho_{0} a^{2}}$.

The Hamiltonian constraint equation

$$
\text { (0) } \begin{gathered}
\Delta \psi=-2 \pi \psi^{5} a^{2}\left[(\rho+P)\left(w^{2}-1\right)+\rho-\rho_{0}\right] \\
-\frac{3 \psi^{5} a^{2}}{16} A^{2},
\end{gathered}
$$

is solved only on the initial time slice with the outer boundary condition $\psi \rightarrow 1+C_{\psi} / 2 r+O\left(r^{-3}\right)$ where $C_{\psi}$ is a constant.

The hydrodynamic equations are written in the form

$$
\begin{gathered}
\partial_{t}\left(w \psi^{6} a^{3} \rho^{1 / \Gamma}\right)+r^{-2} \partial_{r}\left(r^{3} w \psi^{6} a^{3} \rho^{1 / \Gamma} v\right)=0, \\
\partial_{t}\left(w \psi^{6} a^{3}(\rho+P) u\right)+r^{-3} \partial_{r}\left(r^{4} w \psi^{6} a^{3}(\rho+P) v u\right) \\
=-2 \alpha \psi^{6} a^{3} \partial_{y} P+w \psi^{6} a^{3}(\rho+P)\left\{-2 w \partial_{y} \alpha\right. \\
\left.+u\left(\beta+2 y \partial_{y} \beta\right)+\frac{4 \alpha u^{2} y}{\psi^{5} a^{2} w} \partial_{y} \psi\right\},
\end{gathered}
$$

where $v=v^{r} / r$. Using the relation, $\rho_{0}^{1 / \Gamma} a^{3}=$ constant, Eq. (4.8) may be written as

$$
\partial_{t}\left(w \psi^{6} e\right)+r^{-2} \partial_{r}\left(r^{3} w \psi^{6} e v\right)=0,
$$

where $e=\left(\rho / \rho_{0}\right)^{1 / \Gamma}$. In numerical simulation, we choose $D \equiv w \psi^{6} e$ and $S \equiv w \psi^{6}(\rho+P) u$ as variables to be solved. Once $D$ and $S$ are given, $w$ is obtained by solving the algebraic equation

$$
\rho_{0}^{2} \psi^{12} \Gamma^{2}\left(\frac{D}{\psi^{6}}\right)^{2 \Gamma}\left(w^{2}-1\right)=\frac{S^{2} y}{\psi^{4} a^{2}} w^{2 \Gamma-2} .
$$

and $v$ is then given from

$$
\begin{aligned}
v & =-\beta+\frac{\alpha u}{w \psi^{4} a^{2}} \\
& =-\beta+\frac{\alpha S}{\Gamma w^{2} \psi^{10} \rho_{0} a^{2}\left(D / w \psi^{6}\right)^{\Gamma}} .
\end{aligned}
$$

To examine whether a black hole is formed or not, in each time step we search for apparent horizon which is defined as the outermost trapped surface 15. In the spherically symmetric case, the outermost zero point, $r=$ $r_{\mathrm{AH}}$, of the function

$$
\Theta(r) \equiv 2 \frac{\dot{a}}{a}+A+\frac{1}{\psi^{2} a}\left(\frac{2}{r}+\frac{4 \partial_{r} \psi}{\psi}\right),
$$

corresponds to the outermost trapped surface [13]. Hence, we only need to calculate $\Theta$ in each time step and look for the zero point.

Once the apparent horizon is determined, we also calculate the mass of the apparent horizon which we define as

$$
M_{\mathrm{AH}}=\frac{a \psi^{2} r}{2} \text { at } r=r_{\mathrm{AH}} .
$$

$M_{\mathrm{AH}}$ is not identical with the gravitational mass of a black hole in general. However, if it settles down to a constant in the late epoch after formation of the black hole, it can be regarded as the gravitational mass because in the spherical and static space $M_{\mathrm{AH}}$ agrees with the gravitational mass.

In order to check the numerical accuracy of $M_{\mathrm{AH}}$, we also calculate the conserved Kodama mass in the spherical spacetime 116. The Kodama mass within a radius $r$ is defined as

$$
M_{\mathrm{K}}(r)=4 \pi \int_{0}^{r} r^{\prime 2} d r^{\prime} a^{3} \alpha \psi^{6} T_{\mu}^{t} K^{\mu},
$$

where the components of $K^{\mu}$ are

$$
\begin{aligned}
K^{t} & =-\frac{1}{\alpha \psi^{2}} \frac{\partial}{\partial r} \psi^{2} r, \\
K^{r} & =\frac{1}{\alpha \psi^{2}} \frac{\partial}{\partial t} \psi^{2} r
\end{aligned}
$$

and $K^{\theta}=K^{\varphi}=0$. Since $M_{\mathrm{K}}$ at $r=r_{\mathrm{AH}}$ is proved to be equal to $M_{\mathrm{AH}}$ [16], it can be used to check the accuracy of our estimation of $M_{\mathrm{AH}}$. In our simulations, we found that both agree well except for the very late epoch after formation of a black hole at which the gradient of $\alpha$ near the apparent horizon is very steep and it is difficult to keep numerical accuracy well.

Numerical simulations are performed taking 3000 inhomogeneous grid points for the $r$-axis. The circumferential radius of the outer boundary is always kept to be much larger than the Hubble horizon scale. We also take a sufficient number of grids inside a black hole forming region. More concretely, we take grids as $r_{i}=\Delta r\left(f^{i}-1\right) /(f-1)$, where $i=0,1,2, \cdots 3000, f$ is a constant slightly larger than 1 , and $\Delta r$ is the grid spacing at origin which is much smaller than the circumferential length of a formed black hole.

\section{B. Initial conditions}

We make use of the analytic solution derived in Sec. III to give a realistic initial condition for $\rho, u$ and so on. Thus, the initial condition is given at an epoch when the length scale of a density fluctuation is much larger than the Hubble horizon scale.

First, we assume that $\delta=\rho / \rho_{0}-1$ is much smaller than unity and write it as 


$$
\delta=f(r) t^{2-4 / 3 \Gamma} .
$$

From Eq. (3.13), we soon get $\chi=\alpha-1$ as

$$
\chi=-\frac{3 \Gamma-2}{3 \Gamma} f(r) t^{2-4 / 3 \Gamma} .
$$

$u$ is derived from Eq. (4.9) in the long wavelength limit as

$$
u=\frac{2}{3 \Gamma+2} \partial_{y} f(r) t^{3-4 / 3 \Gamma} .
$$

Thus, if we specify the function $f(r)$ on the initial time slice $t=t_{0}$, and subsequently solve Eqs. (4.3) - 4.5) and (4.7), we obtain the initial data for $A, \beta, v, \psi$ and $\alpha$.

In this paper, we simply give $\delta$ as

$$
\begin{gathered}
\delta \cdot \psi^{6}=C_{\delta}\left[\exp \left(-\frac{r^{2}}{r_{0}^{2}}\right)-\sigma^{-3} \exp \left(-\frac{r^{2}}{\sigma^{2} r_{0}^{2}}\right)\right] \\
\times\left(\frac{t}{r_{0}}\right)^{2-4 / 3 \Gamma},
\end{gathered}
$$

where $C_{\delta}, r_{0}$ and $\sigma$ are constants, and we set $a_{f}=r_{0}^{-2 / 3 \Gamma}$. Roughly speaking, $C_{\delta}$ and $\sigma$ specify the amplitude and shape of the density fluctuation, respectively. $r_{0}$ determines the length scale of the density fluctuation, and we fix it to be unity in the following. Hence, hereafter, the mass and length are shown in the units $r_{0}=1$.

If we define the spectrum of density fluctuations as

$$
\delta(k) \equiv \int_{0}^{\infty} j_{0}(k r) \delta \cdot \psi^{6} r^{2} d r,
$$

where $j_{0}(k r) \equiv \sin (k r) / k r$ is the spherical Bessel function of the 0 -th order, we get

$$
\begin{aligned}
\delta(k) & =\frac{\sqrt{\pi} r_{0}^{3}}{4}\left(\frac{t}{r_{0}}\right)^{2-4 / 3 \Gamma} C_{\delta} \\
& \times\left[\exp \left(\frac{-k^{2} r_{0}^{2}}{4}\right)-\exp \left(\frac{-k^{2} r_{0}^{2} \sigma^{2}}{4}\right)\right] .
\end{aligned}
$$

Thus the wavelength of the dominant spectral components of the density fluctuation is larger than $\sim \pi r_{0}$ in the comoving scale (or $k \lesssim 2 / r_{0}$ ). Hence, we start all the simulations at an initial time $t$ which satisfies the condition $t \ll \pi(1-2 / 3 \Gamma) a(t) r_{0}$. In the following, we always set the initial time as $10^{-4} r_{0}$.

Initial conditions are numerically determined by performing iteration as follows: (a) We solve Eq. (4.7) for $\psi$, for the density profile given by Eq. (4.21). (b) From $\delta \cdot \psi^{6}$ as well as $\psi$ obtained in (a), we determine $f(r)$. (c) we calculate $u, w, A$, and $\beta$ by using Eqs. (4.20, $w=\sqrt{1+u^{2} r^{2} \psi^{-4}}$, (4.5) and (4.4), respectively, and substitute the new $w$ and $A$ into Eq. (4.7). We repeat this procedure until sufficient convergence is achieved.

Hereafter, we pay attention only to the $\Gamma=4 / 3$ case, because it is most probable that the universe was radiation-dominated in the early times. In this case, for $t \rightarrow 0$, the metric behaves as

$$
\begin{aligned}
& \chi \rightarrow 0, \\
& \beta \rightarrow \text { const } \ll 1, \\
& \psi-1 \rightarrow \text { const }=O(1) .
\end{aligned}
$$

Apparently, the metric is non-linear for large $C_{\delta}$ at $t=0$ because $\psi-1=O(1)$. Note that $\delta \rightarrow 0$, for $t \rightarrow 0$. Hence, in the present coordinate condition, a black hole is formed only if the metric is non-linear even though the density fluctuation is very small. In other word, we may say that the criterion of formation of black holes depends only on $\psi$ at $t=0$.

\section{Numerical results}

Numerical simulations were performed for various values of $C_{\delta}$ and $\sigma$. Specifically, we surveyed a twodimensional parameter space with $C_{\delta}>0$ and $\sigma>1$. We note that in the case $\sigma=\infty$, the high density peak is surrounded by a flat universe, and in other cases, it is surrounded by a region in which the density is lower than that of the flat universe.

We show the spectrum of $\delta(k)$ for the cases $\sigma=1.5$, $2,3,5,8$ and $\infty$ in Fig. 1. For $\sigma=\infty$, the peak is at $k=0$, while in the other cases, the peak wavenumber and the width around the peak becomes larger and wider, respectively, for smaller $\sigma$.

In Fig. 2, we show $\psi_{0}=\psi(r=0)$ at $t=0$ as a function of $C_{\delta}$ for $\sigma=\infty, 2,3$ and 5 . Here, we can easily calculate $\psi_{0}$ at $t=0$ by using the analytic solution found in Sec. III. It is found that $\psi_{0}$ at $t=0$ monotonically increases with increasing $C_{\delta}$, irrespective of $\sigma$.

In Figs. 3 and 4, we show general features of numerical solutions taking the case $\sigma=\infty$ as an example. The features shown in Figs. 3 and 4 are found also in the cases of finite $\sigma$. In Fig. 3, we show $\delta$ and $2(1-\alpha)$ at origin as a function of time $t$ for black hole formation case $\left(C_{\delta}=15\right.$, dotted lines) and no formation case $\left(C_{\delta}=13\right.$, solid lines), respectively. The reason why we plot $2(1-\alpha)$ for $\alpha$ is that it must coincide with $\delta$ for $t \rightarrow 0$. Fig. 3 clearly shows that for $t \ll r_{0}$, (a) $\delta$ and $\alpha-1$ are proportional to $t$, and (b) $\delta$ agrees with $2(1-\alpha)$. Hence, the numerical solution reproduces the analytic solution for $t \ll r_{0}$ accurately. It is found that the difference between $\delta$ and $2(1-\alpha)$ gradually becomes appreciable at $t / r_{0} \simeq 0.1$ and becomes as large as unity when $t / r_{0} \sim 1$. This is reasonable because the density fluctuation amplitude can become non-linear only after its length scale is smaller than the Hubble horizon scale.

In Fig. 4, we also show $\psi_{0}$ as a function of $t$. As we found in Sec. III, it does not change so much for $t<r_{0}$, but slightly decreases with time evolution. This is due to the effect of the $O\left(\epsilon^{2}\right)$ part in $\psi_{0}$. In some previous works, $\psi_{0}$ at $t \sim r_{0}$ is assumed to be equal to $\psi_{0}$ at $t=0$. 
But as shown here, the assumption is strictly speaking not correct.

In Figs. 5, we show the mass of the apparent horizon $\left(M_{\mathrm{AH}} / r_{0}\right)$ as a function of time $\left(t / r_{0}\right)$ in the black hole formation cases for $\sigma=2,3,5$ and $\infty$. We note that in all the simulations, computation is terminated when it is difficult to keep the numerical accuracy near the apparent horizon. Although the simulations had to be ended before we could draw a definite conclusion, our results given in the figures strongly suggests that $M_{\mathrm{AH}}$ approaches an asymptotic value without increasing forever. This is consistent with a previous result [18, 11. (We note that by comparing it with $M_{\text {Kodama }}$, it is found that $M_{\mathrm{AH}}$ shown here is accurate to within a few percent. )

The formation epoch of a black hole is highly dependent on the initial density profile. In the case $\sigma=\infty$, the formation epoch is $t>100 r_{0}$, but in the other cases, it is earlier for smaller $\sigma$. This is natural because for smaller $\sigma$, the wavenumber of the peak in the spectrum is larger. It is found that $M_{\mathrm{AH}}$ can be $>8 r_{0}$ in the case $\sigma=\infty$, but it is at most $\sim 1.1 r_{0}$ in the case $\sigma=2$. These facts also indicate that the formation epoch and the maximum mass of black holes are highly dependent on the initial density profile. We also note that even when we pay attention only to a particular spectrum shape, $M_{\mathrm{AH}}$ can vary in a large range depending on $C_{\delta}$ (or $\psi_{0}$ at $t=0$ ). Since we do not pursue the simulation in which a black hole in the limit $M_{\mathrm{AH}}=0$ is formed, we cannot draw a strong conclusion, but it seems possible to have a black hole whose mass is much smaller than $r_{0}$ near the threshold of black hole formation.

In Figs. 6, we plot $M_{\mathrm{AH}}$ as a function of $\psi_{0}$ at $t=0$ for the cases $\sigma=2,5$ and $\infty$ as examples. We find that the threshold of $\psi_{0}$ at $t=0$ for formation of black hole is quite different among all the models. In the case $\sigma=\infty$, the threshold value is high $\left(\psi_{0}(t=0) \sim 1.79\right)$. On the other hand, it is not so large in other cases, and smaller for smaller $\sigma$. In Fig. 7, we plot the threshold line on the $\left(\sigma, \psi_{0}(t=0)\right)$-plane. The reason of this property is simple: In the case in which the density peak is surrounded by a high density region, it is forced to be expanded by the surrounding region more strongly and is prevented from collapsing. As a consequence, the density peak surrounded by a higher density region is more difficult to form a black hole, while the density peak surrounded by a lower density region can more easily form a black hole. Probably, the density peak surrounded by a zero density region can most easily form a black hole. Motivated by this observation, we also perform simulations in the flat spacetime from the time symmetric initial condition with the initial density fluctuation profile,

$$
\delta \cdot \psi^{6}=C_{\delta} \exp \left(-\frac{r^{2}}{r_{0}^{2}}\right) .
$$

In this case, the threshold value for $\psi_{0}$ at $t=0$ is about 1.428. This value can be approximately regarded as the smallest value of $\psi_{0}$ at $t=0$ for the formation of a black hole.
As another useful criterion, we point out an approximate measure for determining the formation of black holes in the special case of spherical symmetry. First, by using the Kodama mass, we define an excess mass at each radius at $t=0$ as

$$
\begin{aligned}
\delta M_{\mathrm{K}}(r) & \equiv M_{\mathrm{K}}-M_{\mathrm{F}} \\
& =4 \pi \rho_{0} a^{3} \int_{0}^{r} r^{\prime 2} d r^{\prime} \delta \cdot \psi^{6}\left(1+\frac{2 r^{\prime}}{\psi} \frac{d \psi}{d r}\right),
\end{aligned}
$$

where $M_{\mathrm{F}}$ denotes the mass in the case of a nonperturbed universe and we have used the relations which hold at $t=0$ such as $u=O\left(t^{2}\right), \alpha=1+O(t)$, and $\partial_{t} \psi=O(t)$ to neglect the terms higher order in $t$. Then, we define a compaction function at each radius as

$$
C(r)=\frac{\delta M_{\mathrm{K}}}{r \psi(r)^{2} a} .
$$

Here, we note that this compaction can be defined at $t=0$, because it approaches a time-independent function in the limit $t \rightarrow 0$. In Fig. 8, we show (4.29) as a function of $r$ for some of filled circles on the thick dotted line in Fig. 7. It is found that the maximum value $C(r)_{\max }$ is about 0.4 irrespective of $\sigma$. We note that for each $\sigma$, if the maximum value is large than the value shown in Fig. 8, a black hole is always formed, while if not, it is not. Thus, the measure presented here will be helpful as an approximate criterion to know whether a spherical black hole is formed only from the initial data at $t=0$, and if we use $C(r)_{\max }$ as a parameter instead of $\psi_{0}(t=0)$, we can approximately neglect the dependence on $\sigma$.

\section{SUMMARY}

We have presented a formulation for numerical relativity in the cosmological background by which we can perform a numerical simulation of primordial black hole formation with the initial data that can be analytically given. Namely, we have formulated the Einstein and hydrodynamic equations in the constant mean curvature time-slicing in a way suitable both for obtaining the analytic solution of a perturbation while its length scale is well over the Hubble horizon scale and for performing a numerical simulation until the formation of a black hole. As a result, it becomes possible to investigate the primordial black hole formation from a very early phase of the universe just after inflation up to the formation of black holes in a continuous manner, without changing the gauge condition or numerical matching of the initial data.

By using our formalism, we have carried out a numerical simulation of the black hole formation in a spherically symmetric, radiation-dominated universe, starting from a realistic initial data which are given by the analytical solution of a superhorizon scale perturbation. In this paper, we have considered the initial conditions which are 
specified by two parameters; one characterizes the amplitude of the density (or metric) fluctuation and the other the shape of the density profile. It is found that the formation criterion is moderately dependent on the initial profile of the density fluctuation. In the case when the density peak is surrounded by a flat Friedmann universe, the threshold value of $\psi_{0}$ at $t=0$ for forming a black hole is very large $\sim 1.8$, while when surrounded by a low density region, it may be as small as $\sim 1.4$. This property suggests that the formation of primordial black holes may not be determined by a local criterion: Even when there is a density fluctuation of a high density contrast, it may not efficiently collapse into a black hole if it is in a high density region, but it efficiently collapses if it is in a low density region. As we have noted, this moderate variation of the formation criterion is translated to a very large variation in the actual number of primordial black holes. Thus, we conclude that the spatial correlation of primordial density fluctuations is crucially important in studying the formation of primordial black holes.

In this paper, we have assumed the spherical symmetry and restricted our attention to a model which contains only two parameters. Even in this case, the formation criterion was not so simple. In reality, a primordial black hole is formed in a spacetime without any spatial symmetries. In such a case, the anisotropic effect will be important in addition to the inhomogeneous effect shown in this paper, and it is expected that the formation criterion will be much more complicated than the present case. Apparently, the next step is to carry out non-spherical simulations, and even for such a simulation, the formalism presented here is perfectly applicable.

\section{ACKNOWLEDGMENTS}

We thank K. Nakao for useful discussion and for giving us a note in which Kodama's mass is concisely described. M. Shibata thanks Stu Shapiro for helpful comments. This work was supported in part by the Monbusho Grantin-Aid for scientific research Nos. 08NP0801, 09640355 and 09740336. M. Shibata's work was also supported in part by the JSPS Fellowships for Research Abroad.

[1] For example, Ya. B. Zel'dovich and I. D. Novikov, Astron. Zh. 43, 758 (1966): S. W. Hawking, Mon. Not. R. Astron. Soc. 152, 75 (1971): B. J. Carr and S. W. Hawking, Mon. Not. R. Astron. Soc. 168, 399 (1974): S. W. Hawking, Nature, 248, 30 (1974): D. K. Nadezin, I. D. Novikov, and A. G. Polnarev, Sov. Astron. 22, 129 (1978).

[2] C. Alcock et al. (MACHO collaboration), Astrophys. J. 471, 771 (1996).
[3] For example, R. Kippenhahn and A. Weigert, Stellar Structure and Evolution (Springer, 1990).

[4] T. Nakamura, M. Sasaki, T. Tanaka and K.S. Thorne, Astrophys. J. 487, L139 (1997).

[5] J. Garcia-Bellido, A. Linde and D. Wands, Phys. Rev. D54, 6040 (1996).

[6] J. Yokoyama, Phys. Rev. D58, 083510 (1998).

[7] B. J. Carr, Astrophys. J. 201, 1 (1975).

[8] P. Ivanov, Phys. Rev. D57, 7145 (1998).

[9] H. Kodama and M. Sasaki, Prog. Theor. Phys. supplement 78, 1 (1984).

[10] M. Sasaki and T. Tanaka, Prog. Theor. Phys. 99, 763 (1998).

[11] J.C. Niemeyer and K. Jedamzik, astro-ph/9901292; K. Jedamzik and J.C. Niemeyer, astro-ph/9901293.

[12] M. Shibata and H. Asada, Prog. Theor. Phys. 94, 11 (1995).

[13] M. Shibata, K. Nakao, T. Nakamura and K. Maeda, Phys. Rev. D 50, 708 (1994).

[14] L. Smarr and J. W. York, Phys. Rev. D 17, 1945 (1978).

[15] S. W. Hawking and G. F. R. Ellis, The large scale structure of space-time(Cambridge University Press, 1973).

[16] H. Kodama, Prog. Theor. Phys. 63, 1217 (1980): We thank K. Nakao for giving us a note in which Kodama's result is concisely described.

[17] In particular, for 3D simulation in which the grid number we can take is restricted, the minimum distortion gauge may not be appropriate for a black hole formation problem, because the coordinate spreads outward (i.e., $\beta^{i}>0$ ) when a matter is collapsing (i.e., $v^{i}<0$ ) and hence it is soon difficult to keep the numerical accuracy around the collapsing matter. In the case of 3D simulation, it will be necessary to choose the spatial gauge condition more carefully.

[18] G. V. Bicknell and R. N. Henriksen, Astrophys. J. 232, 670 (1979). 


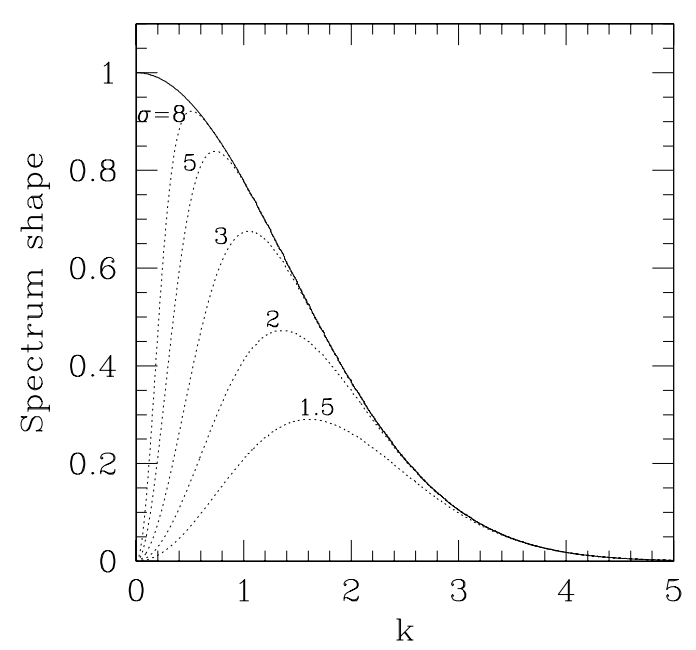

FIG. 1. The spectrum shapes of the density fluctuation $\left(\exp \left(-k^{2} / 4\right)-\exp \left(-k^{2} \sigma^{2} / 4\right)\right)$ are shown for several $\sigma$. The solid line denotes the case for $\sigma=\infty$ in which the density peak is surrounded by a flat universe, and the dotted lines denote the cases for $1.5 \leq \sigma \leq 8$.

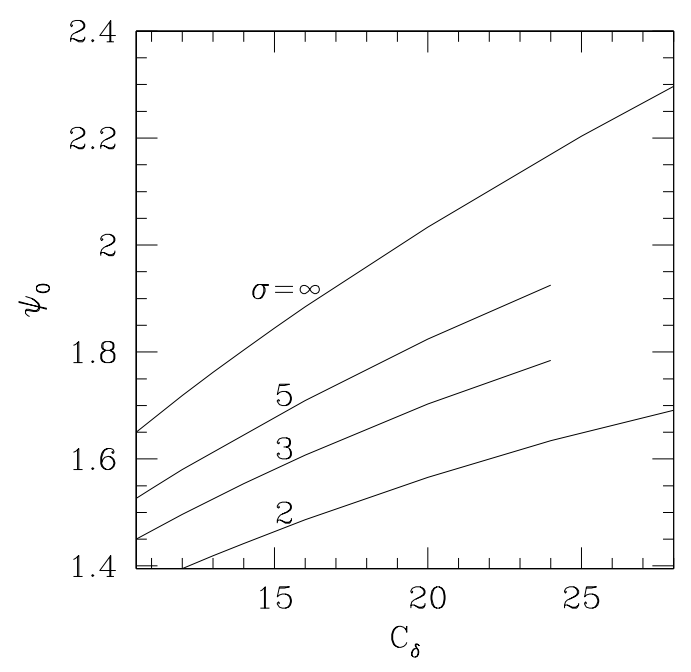

FIG. 2. $\psi_{0}$ at $t=0$ as a function of $C_{\delta}$ for $\sigma=\infty, 2,3$ and

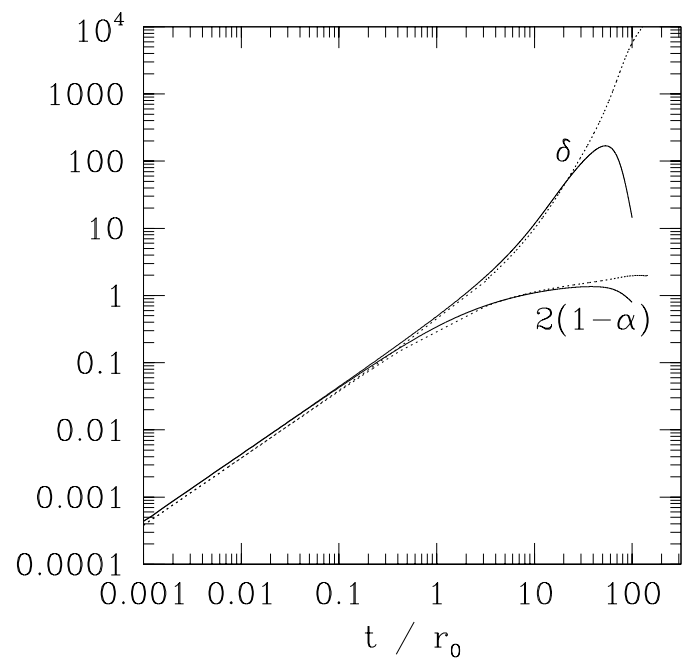

FIG. 3. $\delta$ and $2(1-\alpha)$ at origin as a function of time $t$ for black hole formation case $\left(C_{\delta}=15\right.$, dotted lines) and no formation case $\left(C_{\delta}=13\right.$, solid lines), respectively.

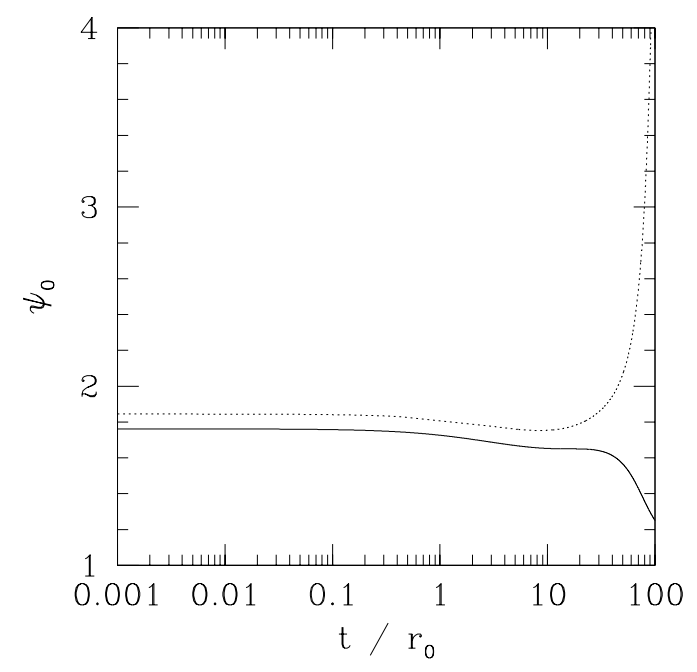

FIG. 4. The same as Fig. 3, but for $\psi_{0}$ as a function of time $t$. 

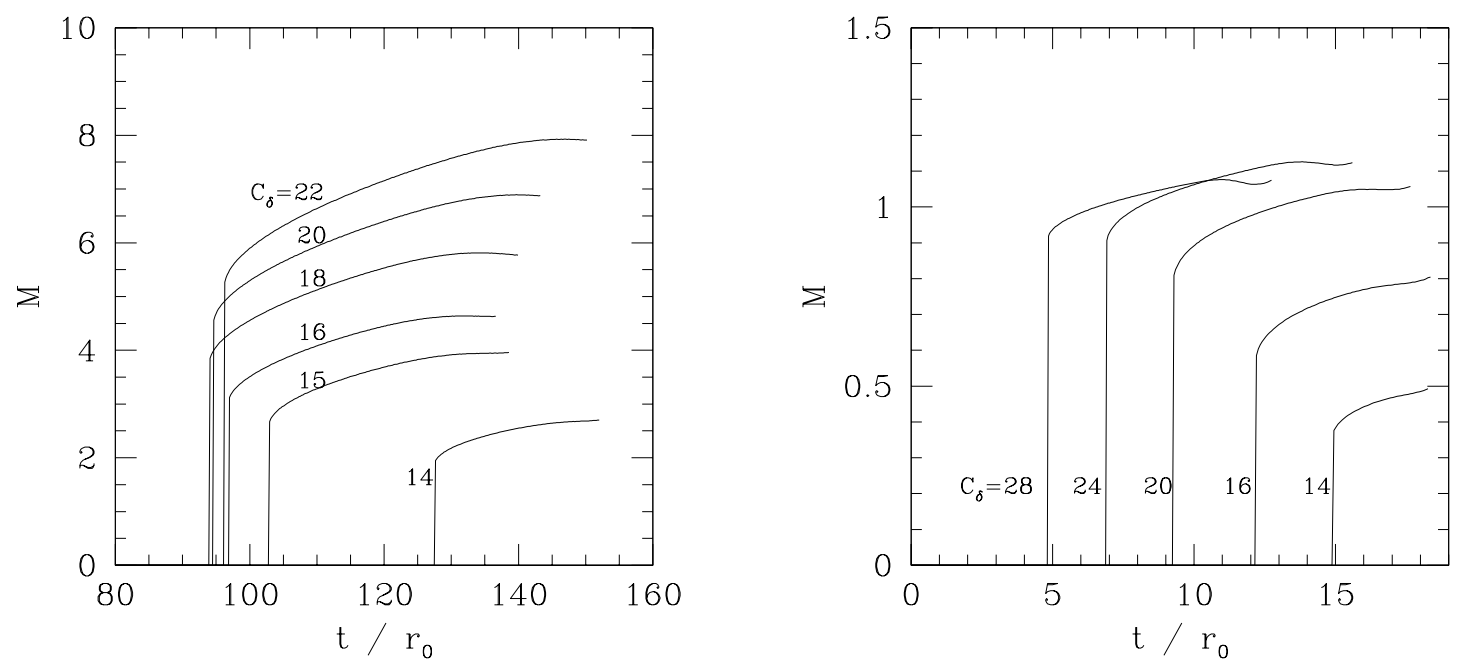

(a)

(b)
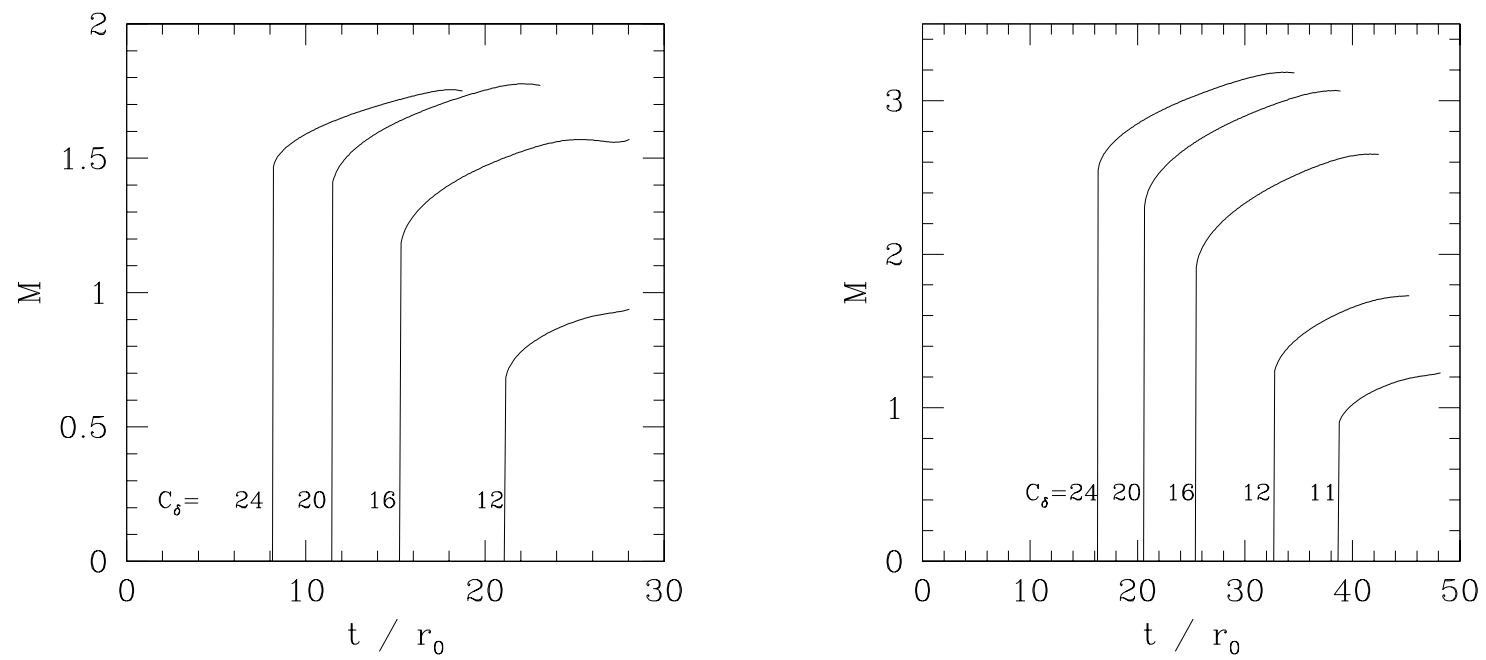

(c)

(d)

FIG. 5. $M_{\mathrm{AH}} / r_{0}$ as a function of time $t / r_{0}$ in black hole formation cases for $\sigma=\infty(\mathrm{a}), 2(\mathrm{~b}), 3(\mathrm{c})$, and $5(\mathrm{~d})$, respectively. 


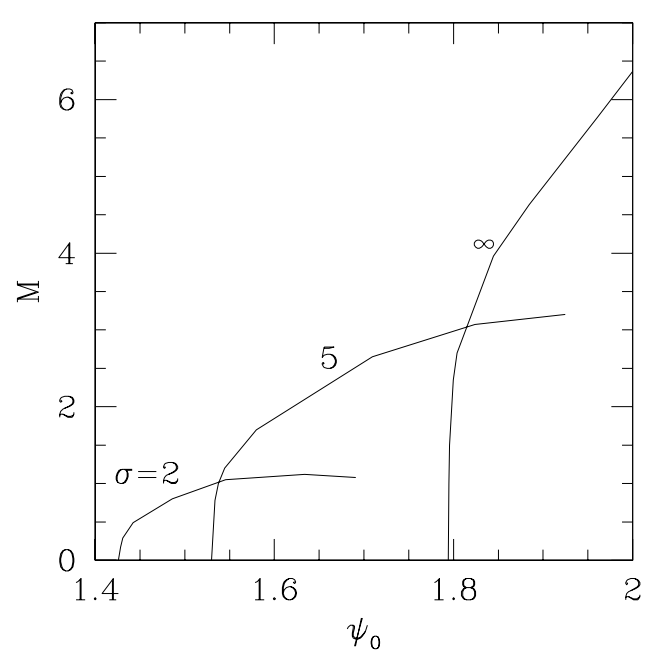

FIG. 6. $M_{\mathrm{AH}} / r_{0}$ as a function of $\psi_{0}$ at $t=0$ for $\sigma=\infty, 2$ and 5 .

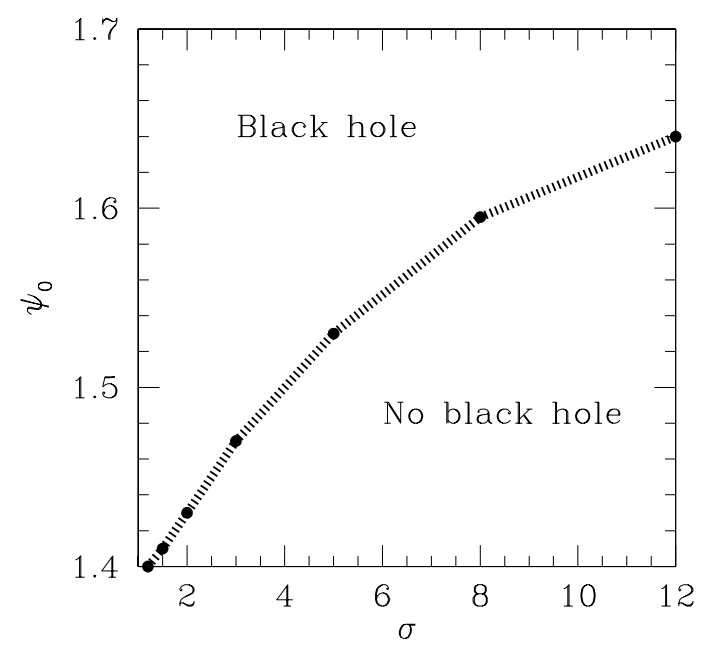

FIG. 7. Summary of numerical results on formation of black holes in $\sigma-\psi_{0}(t=0)$ plane. For initial conditions located in the region above the thick dotted line, we find that a black hole is formed. Note that the solid line reaches $\psi_{0}(t=0) \simeq 1.79$ in the limit $\sigma \rightarrow \infty$.

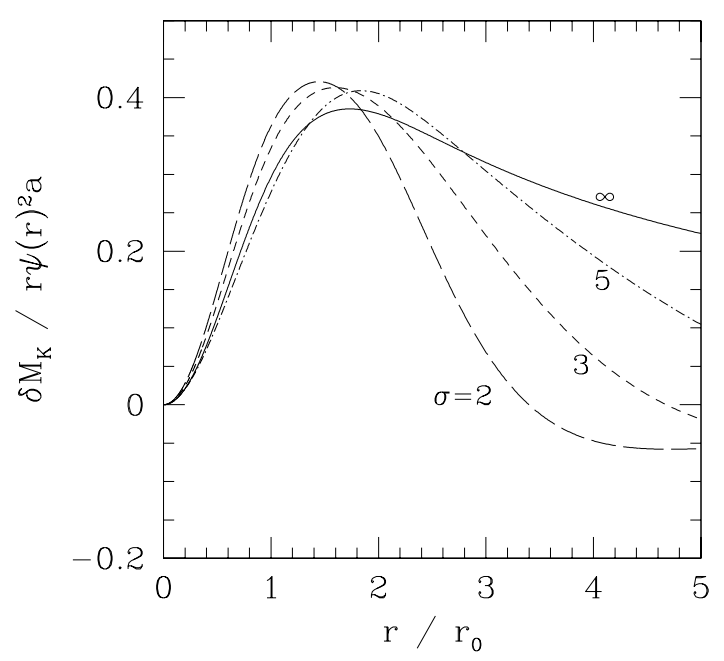

FIG. 8. $\delta M_{\mathrm{K}} / r \psi(r)^{2} a$ as a function of $r$ at $t=0$ for the critical cases of $\sigma=2,3,5$ and $\infty$ (i.e., for the initial condition denoted by filled circles in Fig. 7). The maximum value is $\sim 0.4$ irrespective of $\sigma$. If the maximum value $C(r)_{\max }$ is larger than $\sim 0.4$, a black hole is formed, but if not, it is not for all $\sigma$. 